\title{
Cooperative Interference Cancellation using Device-to-Device Communications
}

\author{
Ralph Tanbourgi, Holger Jäkel and Friedrich K. Jondral \\ Communications Engineering Lab \\ Karlsruhe Insitute of Technology, Germany \\ Contact information: ralph.tanbourgi@kit.edu
}

May 23, 2014

\begin{abstract}
The ongoing densification in cellular networks has turned interference into a serious problem in future cellular networks. Since the interference experienced by close-by users in a cellular network is usually correlated, it can be cooperatively suppressed to improve user experience. In this paper, we introduce the idea of cooperative interference cancellation (CIC) between close-by users using device-to-device (D2D) communications for the example of the upcoming 3rd Generation Partnership Program (3GPP) Long Term Evolution (LTE) Rel-12 D2D technology. We understand CIC as a new network interference management tool, capable of exploiting interference correlation to improve downlink throughput. We discuss possible deployment scenarios as well as theoretical and practical challenges. To each challenge we provide some possible solutions. Finally, a first feasibility analysis using numerical simulations is presented which demonstrates the potential gains of CIC.
\end{abstract}

\section{Introduction and Motivation}

Device-to-Device (D2D) communications has attracted increasing interest in the recent years due to several reasons [1]. This interest, however, first reached only the users, who enthusiastically started exchanging data directly with each other without the need for using the operator's infrastructure. Such short-range communications has been made possible due to the emergence of Bluetooth and WiFi-Direct. After attentively monitoring this trend, operators have now recognized the potentials of D2D as a new business opportunity which led to a dedicated study item within 3GPP LTE Rel-12, in which technical specifications are currently being discussed by the industry [2]. Besides use cases such as context-aware services, authenticated peer-to-peer communications and public safety, which 
are all perceptible to the users, also possible network operation enhancements obtained through user cooperation on the D2D link are being studied [3].

Although still burdened with practical challenges, user cooperation is recently gaining interest also in the cellular community [4], one advantage being to elude the space limitation problem of handhelds preventing the use of many antennas. Historically, user cooperation was understood as a means to solely enhance the information decoding capabilities while ignoring possible commonness in the noise signal. For instance, regenerative relaying for forwarding a de-noised version of the useful signal or maximal ratio combining for optimally boosting the useful signal power. Ignoring the noise properties in the cooperation process was justified by the fact that the decisive source of noise typically lay in the receiver front-end itself, thus rendering noise signals uncorrelated across the cooperating users.

However, by looking at cellular networks today, this perception has clearly become outdated: driven by the ongoing trend of network densification and by opportunistic deployments with limited site-planning, interference has become the performance-limiting factor. In the downlink, interference is caused by other-cell base stations (BSs) transmitting on the same time-frequency resources. In contrast to the noise originating in the front-end of the individual users, the received interference emerges from the same set of BSs, and hence it is correlated across close-by users.

In this article, we introduce the concept of cooperative interference cancellation (CIC) over D2D, which exploits the interference correlation phenomenon between close-by users. In contrast to conventional information-centric relaying, users exchange information regarding correlated interference to improve upon the interference cancellation (IC) process. Since 3GPP D2D is operator-controlled with the possibility of tight control of D2D resources, the CIC concept can be regarded as a new network interference management tool for overcoming inter-cell interference at victim users. To the best of the authors' knowledge, this is the first paper to propose and discuss such a cooperative approach in the context of 3GPP D2D.

Our contributions in this article are structured as follows: Section 2 briefly reviews the idea of (cooperative) IC. The concept of CIC applied to a downlink cellular system using D2D is explained and possible deployment scenarios are discussed. Section 3 provides a comprehensive discussion of the main challenges associated with D2D-based CIC. For each challenge, possible solutions are outlined thereby covering modeling, implementation as well as higher-level issues. Finally, a proof-of-concept is presented in Section 4, where a feasibility analysis is presented using numerical simulations. Section 5 concludes the paper.

\section{Cooperative Interference Cancellation using D2D}

\subsection{Background: Interference Cancellation in Cellular Networks}

Today's cellular networks are designed to operate at high area spectral efficiencies and thus they must face the problem of interference. Although the capacity of cellular networks is yet not fully understood, Shannon's formula $C=W \log _{2}(1+\mathrm{SINR})$ tells us that the rate of a single link can be increased by either choosing a higher transmission bandwidth $W$ or by levering the SINR. In view of spectrum scarcity (a deficit of about $275 \mathrm{MHz}$ by 2014 according to FCC), it is clear that any improvement on the interference control and management side is more than just favorable; and eventually shall bring us closer to the yet unknown capacity of cellular networks.

From an information-theoretic viewpoint, it is desirable to estimate, reconstruct and cancel out the dominant interference from the received signal before decoding the own message. To reliably 

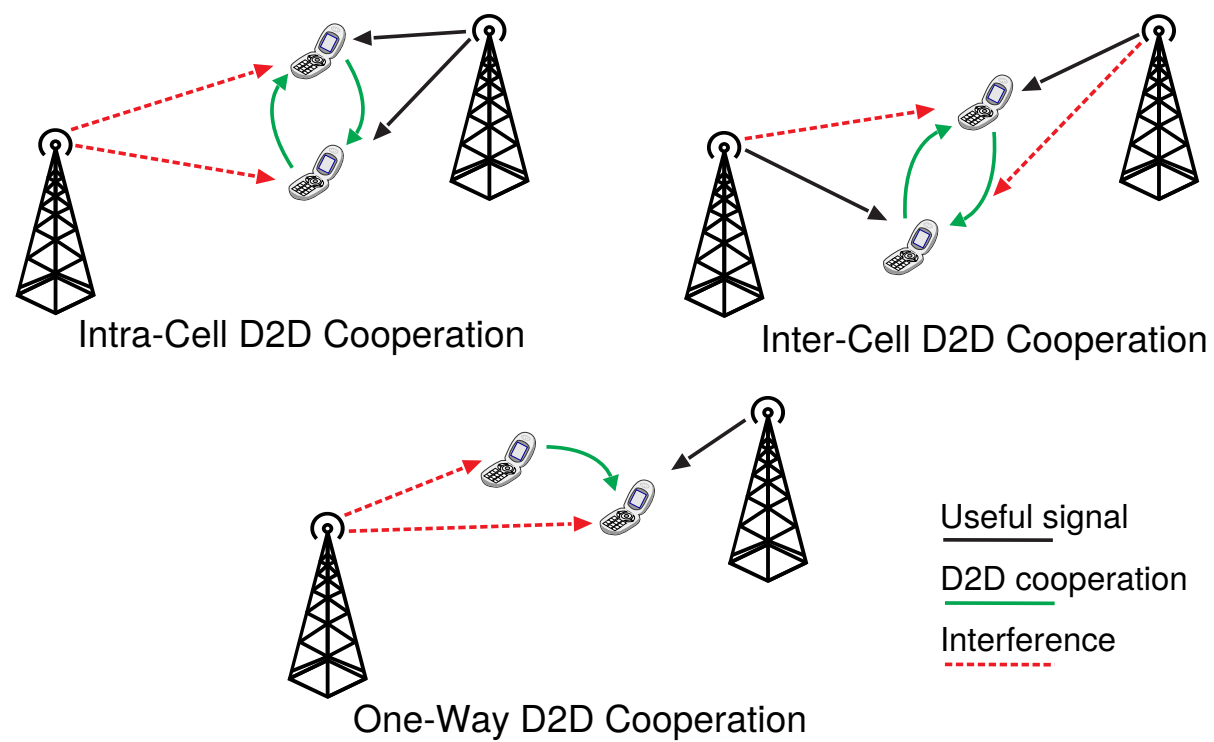

\author{
Useful signal \\ D2D cooperation \\ Interference
}

Figure 1: Illustration of the possible deployment scenarios: a) intra-cell D2D cooperation b), intercell D2D cooperation b) one-way D2D cooperation.

estimate the dominant interference signal, the interference power must be large compared to the residual interference plus noise (interference separability). In the strong interference regime this condition is met and capacity is increased. In contrast, the capacity in the weak interference regime is smaller as the dominant interference signal is cannot be reliably estimated and must be treated as white noise.

IC techniques are expected to become a core component in downlink handheld receivers, see for instance [5] and the references therein. Qualcomm, for example, recently underlined the importance of IC receivers for addressing the thousand-times spectral efficiency challenge. Such advanced receivers are usually equipped with multiple-antennas, which use the additional spatial degrees-offreedom to subtract interference without decoding it; a concept known as spatial IC.

\title{
2.2 Interference Forwarding using D2D
}

For scenarios in which space limitations prevent the use of multiple antennas, IC can still be realized using cooperative methods. A recently studied concept is interference forwarding, where a user forwards the interference signal to the desired receiver. By effectively increasing the interference level, the weak interference regime is transformed into the strong interference regime at the desired receiver, thereby facilitating IC. Alternatively, the forwarded interference signal can be used to spatially cancel out interference from the payload signal like in the case of multi-antenna receivers. It was shown in [6] that interference correlation across the cooperating users is essential for interference forwarding to provide gains.

In cellular networks, CIC can be realized using D2D as the forwarding link. Since 3GPP D2D is operator-controlled, CIC can be employed by the network as an interference management tool. More specifically, a CIC process between a pair of close-by D2D users is triggered by the network whenever 
this pair experiences dominant interference from the same neighboring BS. Using the interference signal forwarded to the victim user, harmful interference can be canceled using a conventional IC approach, e.g., zero-forcing spatial IC. The BS may then choose a higher modulation and coding scheme (MCS) for this user to enhance the downlink throughput.

Before discussing all system aspects involved in this cooperation approach in more detail in Section 3, we first present some possible deployment scenarios.

\subsection{Possible Deployment Scenarios}

The deployment scenarios explained below are illustrated in Fig. 1.

a) Intra-cell D2D cooperation and no inter-cell coordination: Because interference must be correlated for CIC, the cooperative users must receive on the same time-frequency resources in the downlink. Such an allocation is used for instance in multi-user multiple-input multiple-output (MUMIMO) systems. Here, multiple antennas at the BS are used to serve multiple users of the same cell on the same resources with spatially-precoded orthogonally-multiplexed data streams. At the users, the received signal is free from intra-cell interference. Upon reception, the D2D users may then exchange information regarding the observed inter-cell interference to improve the IC process. Note that a competing technique to suppress this kind of interference is downlink coordinated multi-point (CoMP), where inter-cell interference is pre-canceled. A striking advantage of CIC over downlink CoMP, however, is that no low-latency and high-capacity backhaul is required. Backhaul architectures suitable for CoMP are costly and must be pre-deployed in areas where high mobile traffic is expected without the possibility for adaptation. In contrast, CIC using D2D is not tied to a backhaul as it takes places exactly where inter-cell interference is experienced, i.e., locally at the users.

b) Inter-cell D2D cooperation and limited inter-cell coordination: In cellular systems, cell-edge users may suffer from high inter-cell interference. As already mentioned, CoMP techniques can be employed to solve this problem. However, for the reasons outlined before, the capacity of CoMP is usually limited because of imperfect or delayed CSI resulting in non-canceled residual interference. Here, CIC can provide a remedy by additionally post-canceling the residual interference, thereby relaxing the backhaul requirements of CoMP. Furthermore, the required interference correlation across the D2D users can be induced by scheduling two close-by users of different cells on the same radio resources through the existing CoMP backhaul link. Such an intertwining of two IC principles (pre-and post-transmission IC) may help balancing the complexity trade-offs of future cellular networks.

c) One-way D2D cooperation: In this scenario, a user not scheduled for a downlink session agrees upon cooperating with another close-by user. While in conventional relaying, the cooperative user should not forward anything when the channel to the source is in a deep fade, it may now

alternatively forward the observed interference. Such an opportunistic method may then either boost the received useful power or facilitate IC at the victim user.

\section{Challenges And Solutions}

We next outline the challenges associated with D2D-based CIC and provide possible approaches for addressing these challenges. 


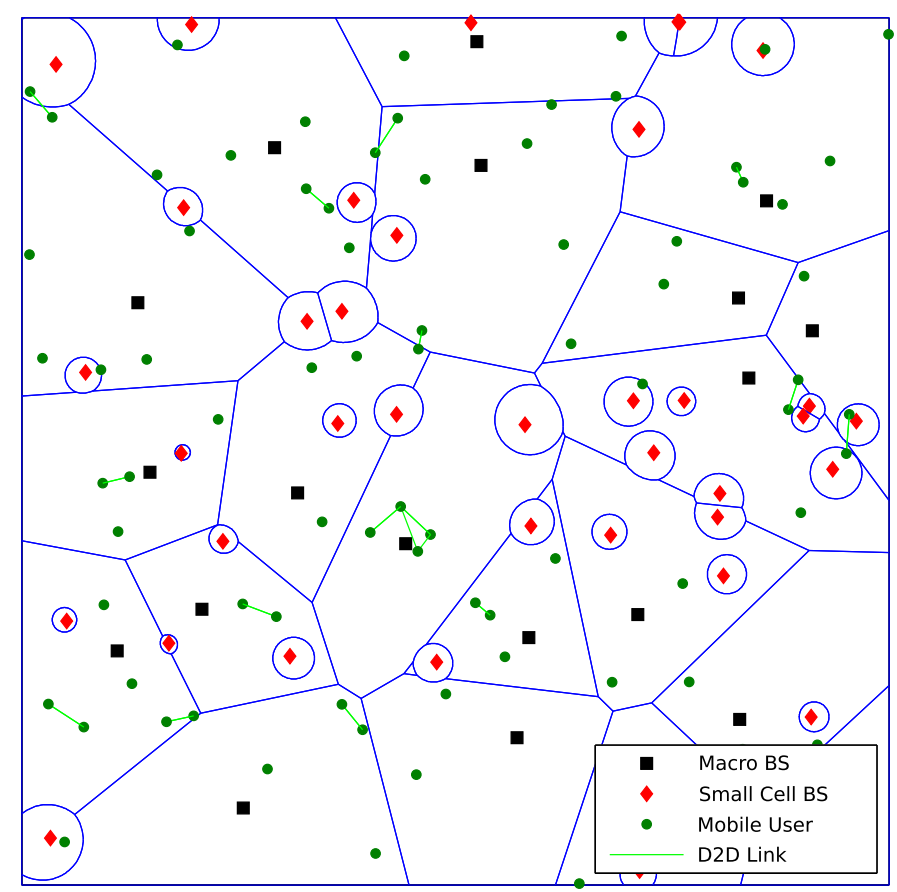

Figure 2: Modeling of a heterogeneous cellular network with D2D links using a Poisson-Voronoi tessellation.

\subsection{Modeling and Analysis Tools}

Tools for analyzing cellular networks play an important role in the development of new techniques. Prominent tools include for instance the Wyner-type models and Monte-Carlo simulations. Cellular networks, however, have recently experienced a substantial technology shift which led to unprecedented issues such as heterogeneity, limited site-planning, irregular deployments and increased mobility, to name just a few. This development has rendered cellular networks opaque and user experience hard-to-predict. In addition, D2D introduces an ad hoc component which makes cellular networks even more complex.

A promising solution to the above challenge of modeling such kinds of networks is the use of stochastic geometry [7]. In principle, the locations of the BSs and the users are modeled by a spatial point process, thereby accounting for irregular deployments, user mobility, and other spatial dynamics. Using these spatial models, key performance metrics such as the SINR or the rate can be analyzed in a comprehensive way. Owing to the many fundamental results available in the literature, e.g., the Campbell theorem or the Laplace functional for Poisson point processes (PPP), closed-form expressions for the key performance metrics can be obtained surprisingly often.

For illustration, Fig. 2 shows an example of a spatial model for heterogeneous two-tier cellular networks with additional D2D users. The two-tier structure was created by independent superposition of two PPPs; the cell-association regions were modeled using a multiplicatively-weighted Poisson-Voronoi tessellation; users were distributed as a PPP and establish a D2D link if sufficiently 


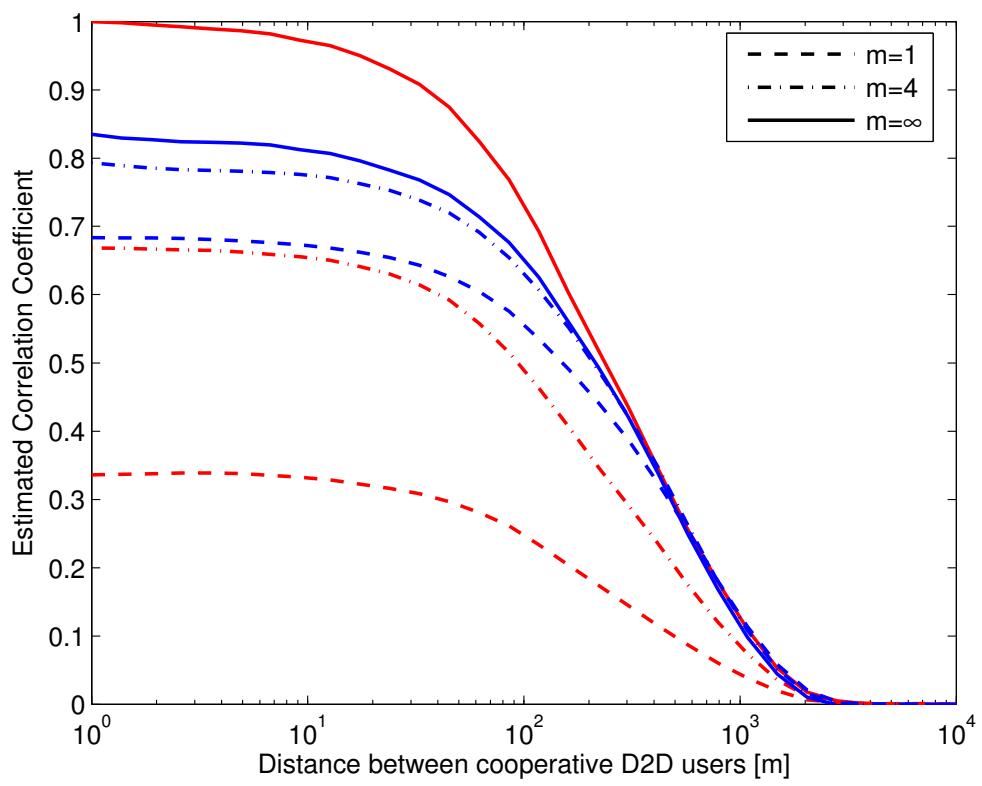

Figure 3: Spatial correlation of the inter-cell interference signal (blue) and power (red) vs. distance between cooperating D2D users. BS locations follow a PPP with density $5 \mathrm{BS} / \mathrm{km}^{2}$. The path loss exponent is $\alpha=4$.

close.

Stochastic geometry can also be used for better understanding the interference correlation phenomenon, which is crucial for CIC to provide gains. Recent works in this field e.g., [8], indicate that interference power in decentralized networks is spatially-correlated over several meters. However, due to the cell-association mechanism, interference correlation decays much slower with distance in cellular networks as illustrated in Fig. 3. In this figure, the estimated interference correlation between two users in a cellular network, where the BSs locations are modeled by a PPP, is shown. For each of the two users, the closest BS is the serving one while interference is caused by intercell BSs. The channel is composed by distance-dependent path loss and Nakagami- $m$ fading with independent and uniformly distributed complex phase. All BSs transmit complex-valued symbols following a circular-symmetric Gaussian distribution with unit-variance. Before correlation, the two signals are aligned with respect to each signal's strongest interferer.

Moreover, it is important to not only look at the correlation of interference powers but also at the correlation of the interference signals. Clearly, correlation of the interference power does not necessarily imply correlation of the interference signals. Hence, a promising research direction could be to characterize the interference signal correlation in cellular networks, possibly using stochastic geometry. 


\subsection{Cooperation Incentives, Resource Allocation and Security}

An important question is whether users are keen to provide their resources (e.g., battery power and degrees-of-freedom) to other users without experiencing an obvious immediate benefit. In order to give users reasons to cooperate, incentives have to be introduced. This may be achieved by using methods from game theory and combining them with stochastic geometry for understanding how cooperation achieving behavior depends on and is limited by the spatial configuration of the users. Exploiting the fact that 3GPP D2D is operator-controlled with the possibility of tight control of D2D resources, all CIC higher-level tasks can be performed by the network rather than by the users themselves. This will bring forward cooperation due to several reasons:

- From the user's side, a fair-minded entity takes care of the cooperation-related decisions, increasing the users' confidence in the cooperation process. This way malice and authentication issues are no longer obstructive to real implementation. Security and privacy can additionally be strengthened through encrypting each user's data at the BSs. In this way, decoding the information bearing data of another user during the cooperation process can be aggravated.

- An operator can boost cooperation by actively promoting it and thereby not relying on decentralized negotiations across the users. Consequently, the number of individual actions are reduced which translates into power and/or degrees-of-freedom savings.

- BSs have much better overview of communications resources, user requirements, channel states etc. Especially, managing the resources used for D2D allocation in the BSs could be part of the BS's scheduling algorithm.

\subsection{Implementation and Design Aspects}

Four aspects are identified to play a key role for designing and implementing CIC in D2D-assisted cellular networks.

Forwarding Scheme: The way cooperation over the D2D interface is achieved must be compliant with link budget constraints. Depending on the the technical constraints, different forwarding strategies are envisaged:

- Amplify-and-Forward (AF): In this scheme, the signal received by the forwarding user is amplified and re-transmitted to the IC user. Importantly, the forwarded signal contains also interference from non-dominant BSs as well as receiver noise which may limit the IC performance. Because the forwarded signal is a scaled version of the received downlink signal, the same time-frequency resources are used which renders this scheme less bandwidth-efficient and non-flexible for resource allocation. While for the underlay D2D mode the latter might not be a problem, this is certainly not true for the overlay D2D mode. Due to its simplicity, $\mathrm{AF}$ is a prominent candidate for CIC and allows for full-duplex operation in principle.

- Decode-and-Forward (DF): Alternatively, the forwarding user can regenerate the dominant interference signal by decoding it, thereby eliminating residual interference and receiver noise. In case the interference signal must be decoded anyways (cf. scenario b) in Section 2.3) this scheme might be appropriate. Otherwise, the receiver complexity is doubled since additional channel estimation and decoding for the dominant interference signal has to be performed. Compared to AF, DF has the advantage that the decoded interference signal (or symbols) do not have to be forwarded on the same time-frequency resources which renders this scheme 
more flexible and compliant with the overlay D2D mode. At the IC user, the forwarded interference signal can be used to zero-force dominant interference in the received downlink signal or to use the decoded interference symbols as side information to facilitate IC.

- Quantize-and-Forward (QF): In this scheme, the interference signal is first quantized at the forwarding node before it is sent to the CIC victim user. The effect of quantization is twofold: first, the received signal is mapped to a low-space representation which reduces the amount of data to be forwarded; second, residual interference and noise is partially eliminated. Similar to DF, this scheme allows for flexible D2D resource allocation and is therefore suitable for both underlay and overlay D2D mode. Especially for the case of nearby cooperating users, QF is considered a efficient forwarding scheme [9].

Synchronization and Estimation: The interference-canceling user has to perform synchronization and estimation tasks in order to reject the dominant interference. In contrast to the organization of medium access between cooperating D2D devices, the cellular network cannot assist this synchronization process at the interference canceling user. Depending on the forwarding scheme, synchronization can be facilitated by (GPS-based) time-stamping of the received signal before forwarding. As demonstrated by Fig. 3, it is already sufficient to align the strongest interferer of each signal to obtain a significant interference correlation. Regarding channel estimation, the fact that reference symbols of neighboring cells are orthogonally-multiplexed in the time-frequency domain can be exploited for estimating the dominant-interference channel. This may be performed either in the time or in the frequency domain owing to the OFDM signal structure of LTE downlink. Hence, the channel estimation and subsequent IC process strongly resembles the conventional zero-forcing multi-antenna receiver principle.

Costs of Cooperation: Besides higher peak data rates and coverage extension, power savings are also anticipated by 3GPP D2D. Therefore, the cooperative methods must work in an energy efficient way in order to not worsen user experience through shorter battery life cycles. The energy used for interference forwarding depends on the bandwidth efficiency of the forwarding schemes as explained above. Furthermore, in the overlay D2D mode, CIC causes a system degrees-of-freedom loss since resources are reserved exclusively for the forwarding process. In case this loss is not acceptable, e.g., in full-load situations, the underlay D2D mode may be more appropriate. Besides, by forwarding the interference signal to the IC user, the cooperative user refrains from transmitting/receiving its own data. Hence, CIC is applicable only to asymmetric traffic situations, e.g., CIC during the uplink phase when little to no data must be conveyed to the BS.

Measuring Interference Correlation: An important point to look at is how to determine the interference correlation at the D2D users and when to trigger a CIC process. One possible approach is outlined as follows: the D2D users feedback CSI that allows the serving BS to identify strong interfering BSs. In LTE, this can be accomplished by letting the users measure the received power to each surrounding BS using the cell-specific reference symbols. If two close-by users share the same dominant interfering BS, this indicates that interference is correlated across the two users. Similarly, interference separability, which is necessary to reliably subtract dominant interference, can be verified in the same way by comparing the interference of the strongest BS to the second strongest or to the residual interference plus receiver noise. 
Table 1: System Parameters used for Simulations

\begin{tabular}{c|c} 
Average inter-BS distance & $500 \mathrm{~m}$ \\
\hline BS output power & $43 \mathrm{dBm}$ \\
\hline Number of subcarriers & 600 \\
\hline Number of OFDM symbols & 7 \\
\hline FFT length & 1024 \\
\hline Subcarrrier spacing & 7 (frequency), 4 (time) \\
\hline Reference symbol spacing & $-162.2 \mathrm{dBW} /$ carrier \\
\hline Thermal noise & Typical Pedestrian \\
\hline Channel & $5 \mathrm{~dB}$ \\
\hline Channel estimation & $23 \mathrm{dBm}$ \\
\hline Noise figure & Least-squares $+2-\mathrm{D}$ interpolation \\
\hline Maximum user transmit power &
\end{tabular}

\section{Feasibility Analysis}

We next present a first comprehensive feasibility analysis of CIC in a D2D-assisted OFDMA cellular system similar to LTE. We consider a ring of eight BSs surrounding a pair of two BSs according to the hexagonal grid model with inter-BS distance of $500 \mathrm{~m}$. A D2D user pair separated by a pre-defined distance is placed on the cell edge between the two inner BSs. To account for irregular BS deployments, the grid model is perturbed by randomly shifting the BSs according to a $2 \mathrm{D}$ zeromean Gaussian distribution with standard deviation $50 \mathrm{~m}$. The two D2D users are served by their closest BS, which is likely to be one of the two BSs inside the ring (other realizations are discarded). We assume that the serving BS performs spatial pre-coding to send orthogonal streams to the two D2D users on the same time-frequency resources, cf. scenario a) in Section 2.3. The subsequent analysis and insights, however, apply in principle also to the other two scenarios introduced in Section 2.3. The system parameters used for the analysis are summarized in Table 1.

\subsection{Considered CIC D2D Scheme}

We consider a typical asymmetric traffic scenario, in which both users wish to receive a large amount of data in the downlink, e.g., a video stream or file download. With some resources left for reliable CSI feedback, we assume that the serving BS can identify (i) the victim user (the one with the lower instantaneous SINR) and (ii) the received interference power caused by other BSs for each of the two users. In case the two users experience dominant interference by the same BS, the serving BS then triggers the following CIC procedure: it selects the appropriate MCS for the non-victim user such that this user can reliably decode the desired signal. Furthermore, the serving BS schedules the non-victim user to perform interference forwarding to the victim user during the uplink phase (half-duplex operation), after having decoded and subtracted the desired signal from the received signal. The decision whether or not to trigger a CIC process depends on the degree of interference separability at the victim user. In this analysis, a CIC process is triggered whenever 


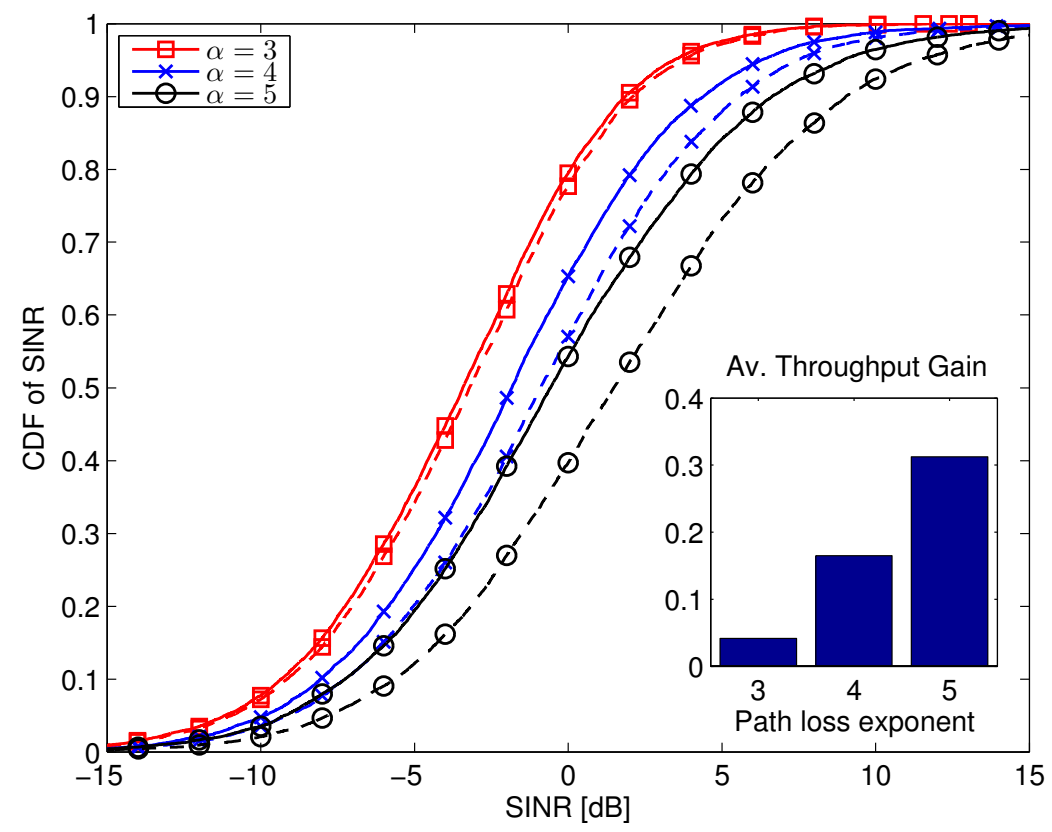

Figure 4: Gains of CIC for a cell-edge scenario. Estimated cumulative distribution function (CDF) of the SINR with (dashed line) and without CIC (solid line) are shown for the victim user for different path loss exponents $\alpha$. The corresponding average throughput gain is shown in the lower right graphic.

the dominant-interference to residual-interference-plus-noise ratio is above 1.5. We further assume an underlay D2D mode and that $\mathrm{AF}$ is used for interference forwarding. At the victim user the two consecutively received and noise-corrupted signals contain correlated interference of the jointly dominant BS. Since reference symbols are orthogonally-multiplexed in the time-frequency domain, the victim user tries to estimate the channels corresponding to the two interference signals from the dominant interferer BS. Afterwards, the channel estimates are used to coherently subtract the dominant interference signal from the received signal containing the desired data. Depending on the channel estimation accuracy (at the users) and the interference separability (verified by the serving BS), the resulting signal has less interference and is then passed through the usual receive chain.

\subsection{First Performance Results}

Figure 4 shows the simulated performance of CIC for the Typical Pedestrian channel model and different path loss exponents $\alpha$ in terms of SINR CDF improvement and average throughput gain. In this scenario, the D2D user separation was set to $1 \mathrm{~m}$ which corresponds to a typical closeproximity situation, e.g., passengers in a bus or customers in a coffee bar. It can be seen that for small $\alpha$, the interference caused by the strongest BS is less dominant. Consequently, a CIC 


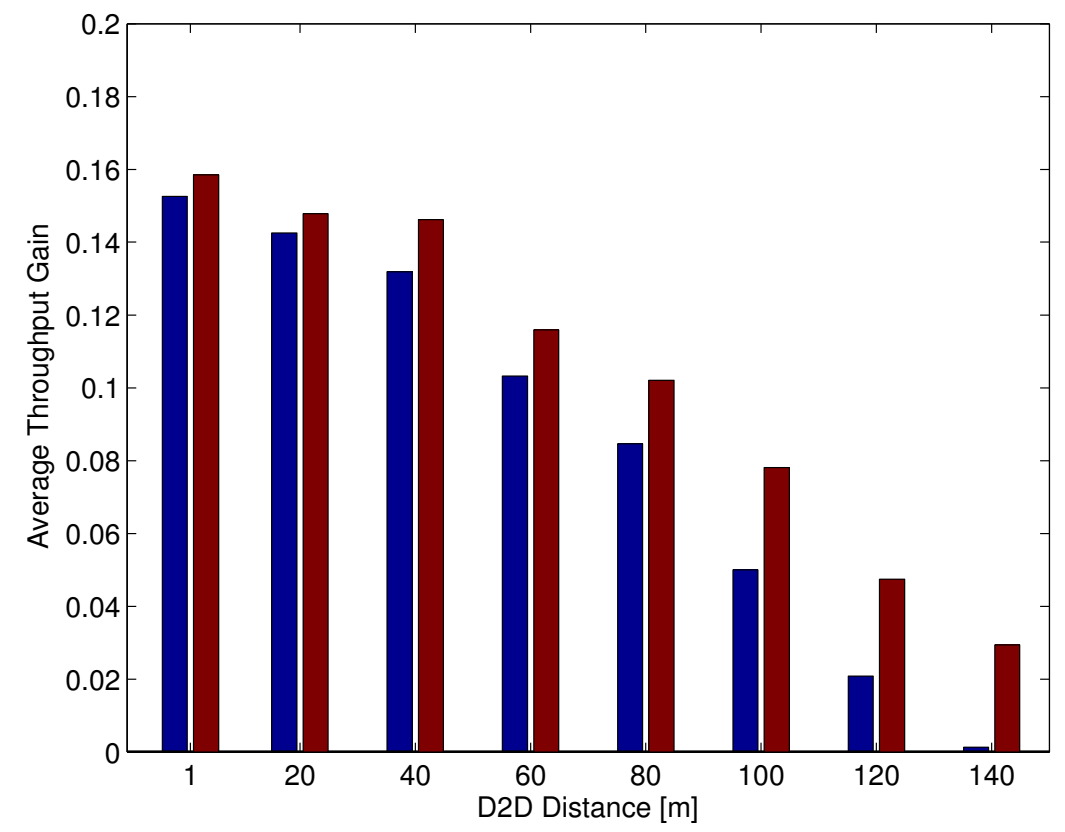

Figure 5: Average throughput gain of CIC vs. D2D-user separation for $\alpha=4$. D2D-user transmit powers are $0 \mathrm{dBm}$ (left) and $5 \mathrm{dBm}$ (right).

procedure is triggered less often resulting in an average throughput gain of about $4 \%$ at $\alpha=3$. With increasing $\alpha$, dominant interference becomes more separable and CIC becomes more beneficial. These gains, however, do not grow unboundedly as receiver noise starts to limit the performance when $\alpha$ becomes very large (not shown here). It can be concluded that the potential gains of CIC are located around $25 \%$ average throughput gain improvement for the typical system parameters summarized in Table 1.

Since interference correlation and link reliability on the D2D link decay with distance, it is important to analyze the resulting performance of CIC when increasing the D2D-user separation. The link reliability is affected by the received D2D signal power (which is assumed to be considerably smaller in the underlay D2D mode to protect the uplink) as well as by interference by other users transmitting in the uplink. In this article, we assume that there is one active user per BS not serving the considered D2D pair. These interfering users reuse the same time-frequency resources as the considered D2D pair and employ power control with full long-term path loss inversion which corresponds to the worst case in terms of interference to the D2D pair.

Figure 5 shows the average throughput gain of CIC vs. the D2D-user separation for different fixed user transmit powers ( $0 \mathrm{dBm}$ left, $5 \mathrm{dBm}$ right). In comparison with Fig. 3, one can see that depending on the allowed transmit power on the D2D link either interference correlation or the link reliability limits the performance of CIC in the underlay D2D mode. 


\section{Conclusion}

In this article, we proposed the concept of CIC using D2D communications, which exploits the spatial interference correlation across close-by users in the downlink. Due to the fact that 3GPP LTE Rel-12 is operator-controlled with the possibility of tight control of D2D resources, we understand this concept as a new network interference management tool to enhance throughput for users with low reception quality. We presented possible deployment scenarios and discussed several design challenges associated with D2D-based CIC. To each of these challenges, we outlined possible solutions. We presented a first feasibility analysis, which confirmed that throughput gains of around $25 \%$ can be achieved with CIC.

\section{Acknowledgment}

This work was supported by the German Research Foundation (DFG) within the Priority Program "COIN" under grant No. JO 258/21-2.

\section{References}

[1] K. Doppler et al., "Device-to-device communication as an underlay to LTE-advanced networks," IEEE Commun. Magazine, vol. 47, no. 12, pp. 42-49, Dec. 2009.

[2] 3GPP, "Technical specification group services and system aspects; feasibility study for proximity services (ProSe) (Release 12)," TR. 22.803, Dec. 2012.

[3] L. Lei, Z. Zhong, C. Lin, and X. Shen, "Operator controlled device-to-device communications in LTE-advanced networks," IEEE Wireless Commun., vol. 19, no. 3, pp. 96-104, Jun. 2012.

[4] F. H. Fitzek, M. Katz, and Q. Zhang, "Cellular controlled short-range communication for cooperative P2P networking," Wirel. Pers. Commun., vol. 48, no. 1, pp. 141-155, Jan. 2009.

[5] J. Andrews, "Interference cancellation for cellular systems: a contemporary overview," IEEE Wireless Commun., vol. 12, no. 2, pp. 19-29, Apr. 2005.

[6] R. Dabora, I. Maric, and A. Goldsmith, "Interference forwarding in multiuser networks," in IEEE Global Telecommun. Conf. (GLOBECOM), 2008, pp. 1-5.

[7] M. Haenggi, Stochastic Geometry for Wireless Networks. Cambridge University Press, 2012.

[8] — , "Diversity Loss due to Interference Correlation," IEEE Commun. Lett., vol. 16, no. 10, pp. 1600-1603, Oct. 2012.

[9] M. Katz and S. Shamai, "Relaying protocols for two colocated users," IEEE Trans. Inform. Theory, vol. 52, no. 6, pp. 2329-2344, Jun. 2006. 